\title{
On Order Invariant Synthesizing Functions
}

\author{
Jean-Luc Marichal \\ Department of Mathematics \\ Brigham Young University, Provo, Utah 84602, USA. \\ Email: marichal@math. byu.edu
}

\begin{abstract}
We give a description of the class of continuous functions that are comparison meaningful in the sense of measurement theory. When idempotency is assumed, this class reduces to the Boolean max-min functions (lattice polynomials). In that case, continuity can be replaced by increasing monotonicity, provided that the domain of definition is open.
\end{abstract}

Keywords: Ordinal scale; Comparison meaningfulness; Ordinal stability; Lattice polynomials.

\section{Introduction}

Consider a set of real numbers defining an ordinal scale, i.e., a scale where only order matters, and not numbers. For example, a scale of evaluation of a scientific paper by a referee such as

$$
\begin{gathered}
1=\text { Poor, } 2=\text { Below Average, } 3=\text { Average }, \\
4=\text { Very Good, } 5=\text { Excellent }
\end{gathered}
$$

is a (finite) ordinal scale, despite the coding by numbers 1 to 5 . These numbers are actually meaningless since any other numbers that preserve the order could have been used. For instance,

$-6.5=$ Poor, $-1.2=$ Below Average, $8.7=$ Average, 205.6=Very Good, 750=Excellent.
Thus, the numbers that are assigned to that scale are defined up to a (continuous) increasing bijection $\phi: \mathbb{R} \rightarrow \mathbb{R}$. For a general discussion of ordinal scales and for definitions of other scale types; see for instance Roberts [17] and Luce et al. [7].

Now, let $x_{1}, \ldots, x_{n}$ be real numbers given according to an ordinal scale. It is clear that any aggregation of these numbers cannot be made by means of usual arithmetic operations, unless these operations involve only order. For example, computing the arithmetic mean is forbidden, but the median or any order statistic is permitted. More precisely, the aggregated value can be calculated only by a synthesizing function $M: \mathbb{R}^{n} \rightarrow \mathbb{R}$ satisfying the following condition:

$$
\begin{gathered}
M\left(x_{1}, \ldots, x_{n}\right)\left\{\begin{array}{r}
\left\{\begin{array}{l}
< \\
=
\end{array}\right. \\
\Downarrow \\
\Downarrow
\end{array} M\left(x_{1}^{\prime}, \ldots, x_{n}^{\prime}\right)\right. \\
M\left(\phi\left(x_{1}\right), \ldots, \phi\left(x_{n}\right)\right)\left\{\begin{array}{l}
< \\
=
\end{array}\right\} M\left(\phi\left(x_{1}^{\prime}\right), \ldots, \phi\left(x_{n}^{\prime}\right)\right)
\end{gathered}
$$

for any $x, x^{\prime} \in \mathbb{R}^{n}$ and any continuous increasing bijection $\phi: \mathbb{R} \rightarrow \mathbb{R}$. Such an order invariant function is said to be comparison meaningful (from an ordinal scale); see Orlov [12].

A typical example of comparison meaningful function is given by the Boolean max-min functions $[8,9,10]$, also called lattice polynomials $[3,14,15]$. These functions are of the form

$$
M(x)=\bigvee_{T \in \mathcal{T}} \bigwedge_{i \in T} x_{i} \quad\left(x \in \mathbb{R}^{n}\right),
$$

where $\mathcal{T}$ is a non-empty family of non-empty subsets of $\{1, \ldots, n\}$. Moreover, symbols $\vee$ 
and $\wedge$ denote maximum and minimum, respectively.

In this paper we described the family of continuous and comparison meaningful functions $M: E^{n} \rightarrow \mathbb{R}$, where $E$ is any real interval, possibly unbounded. When idempotency is assumed (that is, $M(x, \ldots, x)=x$ ), these functions are exactly the Boolean max-min functions. In this case, increasing monotonicity can be substituted to continuity without change.

Note that similar studies have been done for ratio scales and interval scales by Aczél and Roberts [1] and Aczél, Roberts, and Rosenbaum [2].

\section{Boolean max-min functions}

In this section we investigate the Boolean max-min functions, which play a central role in this paper. The word 'Boolean' refers to the fact that these functions are generated by $\{0,1\}$-valued set functions. They are also order invariant extensions on $\mathbb{R}^{n}$ of nonconstant and increasing (monotone) Boolean functions.

To simplify the notation, we set $N:=$ $\{1, \ldots, n\}$.

Definition 2.1 For any non-constant set function $c: 2^{N} \rightarrow\{0,1\}$ such that $c(\emptyset)=0$, the Boolean max-min function $\mathrm{B}_{c}^{\vee \wedge}: \mathbb{R}^{n} \rightarrow \mathbb{R}$ associated to $c$ is defined by

$$
\mathrm{B}_{c}^{\vee \wedge}(x):=\bigvee_{\substack{T \subseteq N \\ c(T)=1}} \bigwedge_{i \in T} x_{i} \quad\left(x \in \mathbb{R}^{n}\right) .
$$

It can be proved [3, Chap. 2, Sect. 5] that any expression constructed from the real variables $x_{1}, \ldots, x_{n}$ and the symbols $\wedge, \vee$ (and, of course, parentheses) is a Boolean max-min function. This shows that the concept of Boolean max-min function is very natural despite its rather strange definition.

Now, we can readily see that any Boolean max-min function $B_{c}^{\vee \wedge}$ fulfills the following property:

$$
\mathrm{B}_{c}^{\vee \wedge}(x) \in\left\{x_{1}, \ldots, x_{n}\right\} \quad\left(x \in \mathbb{R}^{n}\right) .
$$

Actually, we can point out a stronger property. Let $\Pi$ denote the set of all permutations on $N$, and let us introduce the following sets which cover $\mathbb{R}^{n}$ :

$$
\mathcal{O}_{\pi}=\left\{x \in \mathbb{R}^{n} \mid x_{\pi(1)} \leq \cdots \leq x_{\pi(n)}\right\}, \pi \in \Pi .
$$

Clearly, any Boolean max-min function $M=$ $\mathrm{B}_{c}^{\vee \wedge}$ fulfills the following property:

$$
\forall \pi \in \Pi, \exists k \in N \text { s.t. } M(x)=x_{k} \forall x \in \mathcal{O}_{\pi} .
$$

More precisely, we have the following result:

Proposition 2.1 For any $\pi \in \Pi$, we have

$$
\mathrm{B}_{c}^{\vee \wedge}(x)=x_{\pi(j)} \quad\left(x \in \mathcal{O}_{\pi}\right),
$$

with

$$
j=\bigvee_{\substack{T \subseteq N \\ c(T)=1}} \bigwedge_{\pi(i) \in T} i .
$$

The set function $c$ that defines $\mathrm{B}_{c}^{\vee \wedge}$ is not unique. For example, we have

$$
x_{1} \vee\left(x_{1} \wedge x_{2}\right)=x_{1} \quad\left(x_{1}, x_{2} \in \mathbb{R}\right) .
$$

It can be shown [9], however, that there is a unique increasing (monotone) set function $c$ that defines $\mathrm{B}_{c}^{\vee \wedge}$, which is given by

$$
c(T)=\mathrm{B}_{c}^{\vee \wedge}\left(e_{T}\right) \quad(T \subseteq N),
$$

where, for any $T \subseteq N, e_{T}$ is the characteristic vector of $T$ in $\{0,1\}^{n}$.

Using classical distributivity of $\vee$ and $\wedge$, we can see that any Boolean max-min function can also be put in the form:

$$
\bigwedge_{\substack{T \subseteq N \\ d(T)=0}} \bigvee_{i \in T} x_{i}
$$

with an appropriate set function $d: 2^{N} \rightarrow$ $\{0,1\}$, see $[9]$.

Let $f:\{0,1\}^{n} \rightarrow\{0,1\}$ be a non-constant and increasing Boolean function. Then the function $\mathrm{B}_{c}^{\vee \wedge}$, defined with $c(T)=f\left(e_{T}\right)$ $(T \subseteq N)$, is an extension on $\mathbb{R}^{n}$ of $f$ since $f\left(e_{T}\right)=c(T)=\mathrm{B}_{c}^{\vee \wedge}\left(e_{T}\right)$ for all $T \subset N$. Consequently, any Boolean max-min function is an order invariant extension on $\mathbb{R}^{n}$ of a nonconstant and increasing Boolean function. 
Consider now the case of symmetric Boolean max-min functions. For this purpose we recall the concept of order statistic (cf. van der Waerden [18, Sect. 17]).

Definition 2.2 For any $k \in N$, the order statistic function $\mathrm{OS}_{k}: \mathbb{R}^{n} \rightarrow \mathbb{R}$ associated to the $k$ th argument is defined by

$$
\mathrm{OS}_{k}(x)=x_{(k)} \quad\left(x \in \mathbb{R}^{n}\right),
$$

where $(\cdot)$ indicates a permutation on $N$ such that $x_{(1)} \leq \cdots \leq x_{(n)}$.

By Proposition 2.1, we immediately see that any symmetric Boolean max-min function is an order statistic. Conversely, any order statistic is a symmetric Boolean max-min function.

Note that when $n$ is odd, $n=2 k-1$, the particular order statistic $x_{(k)}$ is the well-known median function:

$$
\operatorname{median}\left(x_{1}, \ldots, x_{2 k-1}\right)=x_{(k)} .
$$

Another particular case of Boolean max-min functions is given by the projection functions.

Definition 2.3 For any $k \in N$, the projection function $\mathrm{P}_{k}: \mathbb{R}^{n} \rightarrow \mathbb{R}$ associated to the $k$ th argument is defined by

$$
\mathrm{P}_{k}(x)=x_{k} \quad\left(x \in \mathbb{R}^{n}\right) .
$$

The projection function $\mathrm{P}_{k}$ consists in projecting $x \in \mathbb{R}^{n}$ onto the $k$ th axis. As a particular synthesizing function, it corresponds to a dictatorial aggregation.

\section{Aggregation properties}

Let $E$ be any real interval, bounded or not. Its interior is denoted $E^{\circ}$. The automorphism group of $E$, that is the group of all increasing bijections $\phi: E \rightarrow E$ is denoted by $\Phi(E)$. For the sake of simplicity, we also denote the vector $\left(\phi\left(x_{1}\right), \ldots, \phi\left(x_{n}\right)\right)$ by $\phi(x)$.

In this section we present some aggregation properties that we will use to characterize the set of Boolean max-min functions. The main one is the comparison meaningfulness property, introduced by Orlov [12]. Let us recall its definition.

Definition 3.1 A function $M: E^{n} \rightarrow \mathbb{R}$ is $\Phi$-comparison meaningful ( $\Phi-\mathbf{C M})$ if, for any $\phi \in \Phi(E)$ and any $x, x^{\prime} \in E^{n}$, we have

$M(x)\left\{\begin{array}{l}\stackrel{<}{=} \\ =\end{array} M\left(x^{\prime}\right) \Rightarrow M(\phi(x))\left\{\begin{array}{l}\stackrel{<}{=} \\ =\end{array} M\left(\phi\left(x^{\prime}\right)\right)\right.\right.$.

A stronger requirement is the $\Phi$-ordinal stability, proposed by Marichal and Roubens [11].

Definition 3.2 A function $M: E^{n} \rightarrow E$ is $\Phi$-ordinally stable ( $\Phi$-OS) if, for any $\phi \in$ $\Phi(E)$ and any $x \in E^{n}$, we have

$$
M(\phi(x))=\phi(M(x)) .
$$

The following proposition was proved by Ovchinnikov [13, Theorem 4.1] in the particular case of means; see also [5, 11, 14].

Proposition 3.1 Let the function $M: E^{n} \rightarrow$ E fulfill $\Phi$-OS. Then

$M(x) \in\left\{x_{1}, \ldots, x_{n}\right\} \cup\{\inf E, \sup E\} \quad\left(x \in E^{n}\right)$.

Furthermore, if $E$ is open then

$$
M(x) \in\left\{x_{1}, \ldots, x_{n}\right\} \quad\left(x \in E^{n}\right) .
$$

In the second part of Proposition 3.1, the assumption that $E$ is open is necessary. Indeed, if $a:=\inf E \in E$ for example, then any $\phi \in \Phi(E)$ is such that $\phi(a)=a$ and thus the constant function $M=a$ fulfills $\Phi$-OS.

The most often encountered synthesizing functions in the literature on aggregation are means or averaging functions, such as the weighted arithmetic means. Cauchy [4] considered in 1821 the mean of $n$ independent variables $x_{1}, \ldots, x_{n}$ as a function $M\left(x_{1}, \ldots, x_{n}\right)$ which should be internal to the set of $x_{i}$ values.

Definition 3.3 A function $M: E^{n} \rightarrow \mathbb{R}$ is internal (Int) if

$\min x_{i} \leq M\left(x_{1}, \ldots, x_{n}\right) \leq \max x_{n}\left(x \in E^{n}\right)$. 
Such means satisfy trivially the property of idempotency, i.e., if all $x_{i}$ are identical, $M(x)$ restitutes the common value.

Definition 3.4 A function $M: E^{n} \rightarrow \mathbb{R}$ is idempotent (Id) if

$$
M(x, \ldots, x)=x \quad(x \in E) .
$$

The characterizations we will present in the next section are mainly devoted to idempotent functions. We shall also use two other aggregation properties: continuity $(\mathbf{C o})$ and increasing monotonicity in each argument (In).

The Id property seems natural enough, even when values to be aggregated are defined on an ordinal scale. Besides, one can readily see that, for functions fulfilling $\mathbf{I n}$, it is equivalent to Cauchy's internality Int, and both are accepted by all statisticians as requisites for means and typical values.

The following result, adapted from Lemma 2.2 in [13], shows that ordinal stability and comparison meaningfulness are closely related properties.

Proposition 3.2 Consider a function $M: E^{n} \rightarrow E$.

i) If $M$ fulfills Id and $\Phi-\mathbf{C M}$ then it fulfills $\Phi$-OS.

ii) If $M$ fulfills $\Phi$-OS then it fulfills $\Phi$-CM.

iii) If $E$ is open then $M$ fulfills Id and $\Phi-\mathbf{C M}$ if and only if it fulfills $\Phi-\mathbf{O S}$.

\section{Main results}

In the present section we give the axiomatic characterizations stated in the introduction.

Theorem 4.1 The function $M: E^{n} \rightarrow \mathbb{R}$ fulfills Co and $\Phi-\mathbf{C M}$ if and only if

- either $M$ is constant,

- or there exists a set function $c$ and a continuous and strictly monotonic function $g: E \rightarrow \mathbb{R}$ such that $M=g \circ \mathrm{B}_{c}^{\vee \wedge}$.

The following two characterizations follow immediately from Theorem 4.1; see also [10].
Corollary 4.1 The function $M: E^{n} \rightarrow \mathbb{R}$ fulfills $\mathbf{C o}$, Id, and $\Phi-\mathbf{C M}$ if and only if there exists a set function $c$ such that $M=\mathrm{B}_{c}^{\vee \wedge}$.

Corollary 4.2 Assume that $E$ is open. Then the function $M: E^{n} \rightarrow E$ fulfills Co and $\Phi$ OS if and only if there exists a set function $c$ such that $M=\mathrm{B}_{c}^{\vee \wedge}$.

Note that Corollary 4.2 was stated and proved by Ovchinnikov [14, Theorem 5.3] in the more general setting where the range of variables is a doubly homogeneous linear order (i.e., a set $X$ fulfilling the following property: for any $x_{1}, x_{2}, y_{1}, y_{2} \in X$, with $x_{1}<x_{2}$ and $y_{1}<y_{2}$, there is an automorphism $\phi: X \rightarrow X$ such that $\phi\left(x_{1}\right)=y_{1}$ and $\left.\phi\left(x_{2}\right)=y_{2}\right)$.

We have already observed in the remark regarding Proposition 3.1 that, when $E$ is not open, there exist functions $M: E^{n} \rightarrow E$ fulfilling Co and $\Phi$-OS other than $\mathrm{B}_{c}^{\vee \wedge}$. The complete description of that family is given in the following result.

Corollary 4.3 The function $M: E^{n} \rightarrow E$ fulfills Co and $\Phi$-OS if and only if

- either $M=\inf E$ (unless inf $E \notin E$ ),

- or $M=\sup E$ (unless $\sup E \notin E$ ),

- or there exists a set function c such that $M=\mathrm{B}_{c}^{\vee \wedge}$.

It follows from Corollary 4.3 that the functions $M: E^{n} \rightarrow E$ that fulfill Co, Id, and $\Phi$-OS are exactly the Boolean max-min functions. The extension of this latter result to the case of functional operators can be found in Ovchinnikov and Dukhovny [16].

Now, let us turn to the case of increasing functions. We have the following result; see also [10].

Theorem 4.2 Assume that $E$ is open. Then the function $M: E^{n} \rightarrow \mathbb{R}$ fulfills $\mathbf{I n}, \mathbf{I d}$, and $\Phi-\mathbf{C M}$ if and only if there exists a set function c such that $M=\mathrm{B}_{c}^{\vee \wedge}$.

If $E$ is not open, the set of Boolean max-min functions on $E^{n}$ cannot be characterized by the properties In, Id, and $\Phi$-CM. For example, the function $M^{*}:[a, b]^{n} \rightarrow \mathbb{R}$, defined 
by

$$
M^{*}(x)= \begin{cases}b, & \text { if } \max _{i} x_{i}=b, \\ \min _{i} x_{i}, & \text { else, }\end{cases}
$$

fulfills these three properties, but is not a Boolean max-min function on $[a, b]^{n}$.

Theorem 4.2 shows that the discontinuities as in (1) occur only on the border of $E^{n}$. Thus, any function $M: E^{n} \rightarrow \mathbb{R}$ fulfilling $\mathbf{I n}, \mathbf{I d}$, and $\Phi-\mathbf{C M}$ is a Boolean max-min function on $\left(E^{\circ}\right)^{n}$.

Corollary 4.4 Assume that $E$ is open. Then the function $M: E^{n} \rightarrow E$ fulfills In and $\Phi$ $\mathrm{OS}$ if and only if there exists a set function $c$ such that $M=\mathrm{B}_{c}^{\vee \wedge}$.

\section{Order statistics and projection functions}

We now intend to characterize the order statistics and the projection functions, which are particular Boolean max-min functions.

Since the order statistics are exactly the symmetric Boolean max-min functions, we immediately have the following three characterizations. The notation Sy stands for the symmetry property.

Corollary 5.1 The function $M: E^{n} \rightarrow \mathbb{R}$ fulfills $\mathbf{S y}$, Co, and $\Phi-\mathbf{C M}$ if and only if

- either $M$ is constant,

- or there exists $k \in N$ and a continuous and strictly monotonic function $g: E \rightarrow \mathbb{R}$ such that $M=g \circ \mathrm{OS}_{k}$.

Corollary 5.2 The function $M: E^{n} \rightarrow \mathbb{R}$ fulfills $\mathbf{S y}, \mathbf{C o}$, Id, and $\Phi-\mathbf{C M}$ if and only if there exists $k \in N$ such that $M=\mathrm{OS}_{k}$.

Corollary 5.3 Assume that $E$ is open. Then the function $M: E^{n} \rightarrow \mathbb{R}$ fulfills $\mathbf{S y}, \mathbf{I n}, \mathbf{I d}$, and $\Phi-\mathbf{C M}$ if and only if there exists $k \in N$ such that $M=\mathrm{OS}_{k}$.

Note that the second characterization, when Int replaces Id, was proved first by Orlov [12] on $\mathbb{R}^{n}$, then by Marichal and Roubens [11, Theorem 1] on $E^{n}$, and finally by Ovchinnikov [13, Theorem 4.3] in the more general framework of ordered sets. The two other characterizations were previously unknown.

Now, let us characterize the median function, which is a particular order statistic. For that purpose we introduce the following property.

Definition 5.1 Let $\psi: E \rightarrow E$ be a decreasing bijection. A function $M: E^{n} \rightarrow \mathbb{R}$ is stable with respect to a $\psi$-reversal of the scale $\left(\psi\right.$-SR) if for any $x, x^{\prime} \in E^{n}$, we have

$M(x)=M\left(x^{\prime}\right) \quad \Rightarrow \quad M(\psi(x))=M\left(\psi\left(x^{\prime}\right)\right)$, where the notation $\psi(x)$ means $\left(\psi\left(x_{1}\right), \ldots, \psi\left(x_{n}\right)\right)$.

We then have the following results.

Corollary 5.4 Assume that $n$ is odd. There exists a decreasing bijection $\psi: E \rightarrow E$ such that the function $M: E^{n} \rightarrow \mathbb{R}$ fulfills $\mathbf{S y}$, Co, $\Phi-\mathbf{C M}$, and $\psi$-SR if and only if

- either $M$ is constant,

- or there exists a continuous and strictly monotonic function $g: E \rightarrow \mathbb{R}$ such that $M=g \circ$ median.

Corollary 5.5 Assume that $n$ is odd. There exists a decreasing bijection $\psi: E \rightarrow E$ such that the function $M: E^{n} \rightarrow \mathbb{R}$ fulfills $\mathbf{S y}$, Co, Id, $\Phi-\mathbf{C M}$, and $\psi$-SR if and only if $M=$ median.

Corollary 5.6 Assume that $E$ is open and that $n$ is odd. There exists a decreasing bijection $\psi: E \rightarrow E$ such that the function $M: E^{n} \rightarrow \mathbb{R}$ fulfills $\mathbf{S y}, \mathbf{I n}, \mathbf{I d}, \Phi-\mathbf{C M}$, and $\psi$-SR if and only if $M=$ median.

Now, let us turn to the case of projection functions. As we can easily see, the projection functions fulfill the following property $[1,6]$.

Definition 5.2 A function $M: E^{n} \rightarrow \mathbb{R}$ is $\Phi$-comparison meaningful from independent ordinal scales (Ф-CMI) if, for any $\phi_{1}, \ldots, \phi_{n} \in \Phi(E)$ and any $x, x^{\prime} \in E^{n}$, we have

$M(x)\left\{\begin{array}{l}< \\ =\end{array}\right\} M\left(x^{\prime}\right) \Rightarrow M(\phi(x))\left\{\begin{array}{l}< \\ =\end{array}\right\} M\left(\phi\left(x^{\prime}\right)\right)$, where the notation $\phi(x)$ means $\left(\phi_{1}\left(x_{1}\right), \ldots, \phi_{n}\left(x_{n}\right)\right)$. 
It can be shown that the projection functions are exactly those Boolean max-min functions which fulfill $\Phi$-CMI. We then have the following results.

Corollary 5.7 The function $M: E^{n} \rightarrow \mathbb{R}$ fulfills Co and $\Phi-\mathbf{C M I}$ if and only if

- either $M$ is constant,

- or there exists $k \in N$ and a continuous and strictly monotonic function $g: E \rightarrow \mathbb{R}$ such that $M=g \circ \mathrm{P}_{k}$.

Corollary 5.8 The function $M: E^{n} \rightarrow \mathbb{R}$ fulfills Co, Id, and $\Phi$-CMI if and only if there exists $k \in N$ such that $M=\mathrm{P}_{k}$.

Corollary 5.9 Assume that $E$ is open. Then the function $M: E^{n} \rightarrow \mathbb{R}$ fulfills $\mathbf{I n}, \mathbf{I d}$, and $\Phi-\mathbf{C M I}$ if and only if there exists $k \in N$ such that $M=\mathrm{P}_{k}$.

\section{References}

[1] J. Aczél and F.S. Roberts, On the possible merging functions, Math. Social Sciences 17 (1989) 205-243.

[2] J. Aczél, F.S. Roberts and Z. Rosenbaum, On scientific laws without dimensional constants, Journal of Math. Analysis and Appl. 119 (1986) 389-416.

[3] G. Birkhoff, Lattice Theory, (Third Edition, AMS, Providence, 1967).

[4] A.L. Cauchy, Cours d'analyse de l'Ecole Royale Polytechnique, Vol. I. Analyse algébrique, (Debure, Paris, 1821).

[5] J.C. Fodor and M. Roubens, On meaningfulness of means, Journal of Computational and Applied Mathematics 64 (1995) 103-115.

[6] S.-R. Kim, On the possible scientific laws, Mathematical Social Sciences 20 (1990) 19-36.

[7] R.D. Luce, D.H. Krantz, P. Suppes and A. Tversky, Foundations of measurement, volume III, (Academic Press, New York, 1990).
[8] J.-L. Marichal, Aggregation operators for multicriteria decision aid, Ph.D. Thesis, University of Liège, Liège, Belgium, 1998.

[9] J.-L. Marichal, On Sugeno integral as an aggregation function, Fuzzy Sets and Systems 114 (3) (2000) 347-365.

[10] J.-L. Marichal and P. Mathonet, On comparison meaningfulness of aggregation functions, Journal of Mathematical Psychology 45 (2) (2001) 213-223.

[11] J.-L. Marichal and M. Roubens, Characterization of some stable aggregation functions, in: Proc. Intern. Conf. on Industrial Engineering and Production Management, Mons, Belgium, June 2-4, 1993, pp. 187-196.

[12] A. Orlov, The connection between mean quantities and admissible transformations, Mathematical Notes 30 (1981) 774-778.

[13] S. Ovchinnikov, Means on ordered sets, Mathematical Social Sciences 32 (1996) $39-56$.

[14] S. Ovchinnikov, Invariant functions on simple orders, ORDER 14 (1998) 365371.

[15] S. Ovchinnikov, On ordinal OWA operators, 8th Int. Conf. on Information Processing and Management of Uncertainty in Knowledge-Based Systems (IPMU'98), Paris, 1998.

[16] S. Ovchinnikov and A. Dukhovny, On order invariant aggregation functionals, Journal of Mathematical Psychology, to appear.

[17] F.S. Roberts, Measurement theory with applications to decision-making, utility and the social sciences, (Addison-Wesley Pub., Reading, MA, 1979).

[18] B.L. van der Waerden, Mathematical statistics, (Springer-Verlag, Berlin, 1969). 